Ahmad, I. and Khawaja, E. E.

\title{
Passive cooling of surfaces
}

\author{
Ahmad, I.* and Khawaja, E. E. \\ Department of Physics, School of Science, \\ University of Management and Technology, \\ C-II Johar Town, Lahore, Pakistan \\ *imtiaz.ahmad@umt.edu.pk,
}

\begin{abstract}
Radiative cooling of surfaces without applying an energy source has been suggested in the literature. The basis for radiative cooling is the infrared transmittance of the atmosphere. The spectral transparency (i.e. low absorption) of the atmosphere in the wavelength range of 8 to $13 \mu \mathrm{m}$ is generally termed the atmospheric window. If the humidity and cloudiness are low, then the downward thermal radiation from the atmosphere in the spectral region of the atmospheric window are expected to be low. A temperature between $15^{\circ} \mathrm{C}$ and $25^{\circ} \mathrm{C}$ below ambient temperature has been achieved as a result of radiative cooling.

Spectrally selective radiating material, which is defined as a material with high emittance in the spectral region of the atmospheric window and high reflectance in the rest of the spectral range, can be used as an essential means in a sky radiator for passive cooling of the material. Silicon monoxide film on highly reflective substrate (aluminum coated glass) was used in the present work. It was found that the temperature of the thin film surface was about $12{ }^{\circ} \mathrm{C}$ lower than that of ambient temperature under suitable condition of radiative cooling. Cooling is expected to be more efficient in performance if it is carried out in desert places.
\end{abstract}

Keywords: thin films, transmittance, radiative cooling, silicon monoxide

\section{Introduction}

A passive cooling strategy works without applying input electricity. In order to achieve passive cooling below ambient temperature at night, a method known as radiative cooling has been established [1-4]. The basis for the radiative cooling is the infrared transmittance of the atmosphere [5]. The spectral transparency (i.e. low absorption) of the atmosphere in the wavelength range of 8 to $13 \mu \mathrm{m}$ is generally termed the atmospheric window. It is obvious that most of the energy transfer occurs through this window due to the radiative cooling [6-8]. On the other hand, thermal radiation absorbed by carbon dioxide and water vapor in the atmosphere also results in the discharge of the thermal radiation downwards from the atmosphere. It has been reported [9] that radiative cooling by spectral selective 
radiative material (SSRM) appears most significant under certain weather conditions which are low or moderate cloudiness and humidity. Due to the radiative cooling, a temperature from $15{ }^{\circ} \mathrm{C}$ to $25{ }^{\circ} \mathrm{C}$ which is below ambient temperature may be achieved by a blackbody. Spectral selective radiative materials have a high emittance (low reflectance) in the spectral region of the atmospheric window and a high reflectance in the remaining spectral ranges [10-14]. In this present work, silicon monoxide $(\mathrm{SiO})$ films were deposited on highly reflective substrate ( $\mathrm{Al}$ on glass). Silicon based films are characterized as one of the most suitable material in SSRM system. Earlier similar work was performed in various countries other than Saudi Arabia. The present work was performed in Dhahran, Saudi Arabia.

\section{Experimental Details}

In this work, a two layered system on glass of $\mathrm{SiO} / \mathrm{Al} /$ glass was fabricated by using physical vapor method (PVD). A tungsten boat was used for evaporation of $\mathrm{SiO}$, whereas Al films were prepared by electron beam evaporation. Purity of the materials used for evaporation were better than $99.9 \%$. The thickness of the Al layer was about $100 \mathrm{~nm}$. The thickness of the $\mathrm{SiO}$ layer varied between 800 to $1500 \mathrm{~nm}$. Thickness greater than about $1000 \mathrm{~nm}$ were good enough. After deposition, the $\mathrm{SiO} / \mathrm{Al} / \mathrm{glass}$ device was placed in a polystyrene block as shown in figure 1 . The device was covered with a black polyethylene sheet. The black sheets provide insulation against convective heat inflow and prevent the SiO layer from visible and near infrared radiation generated by streetlights and glow in the sky. A thermocouple was connected to the device while another was left to measure the atmospheric temperature.

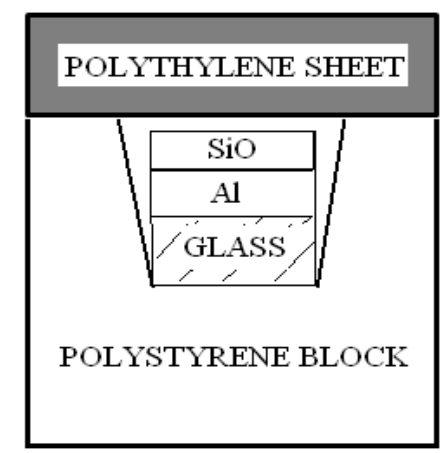

Figure 1: Cross-sectional view of a panel for testing radiative cooling

\section{Results and Discussion}

Figure 2 shows the measured spectral transmittance (T) of a $\mathrm{SiO}$ film on $\mathrm{KBr}$ substrate in infrared region.

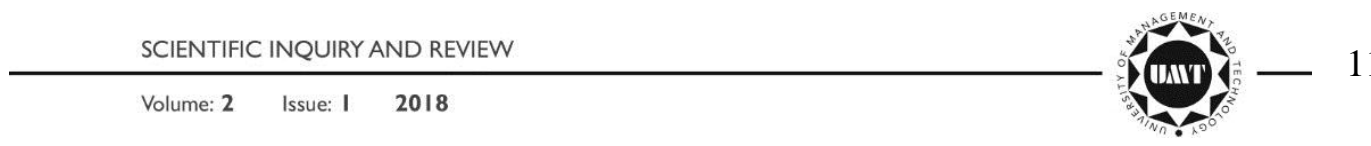


Ahmad, I. and Khawaja, E. E.

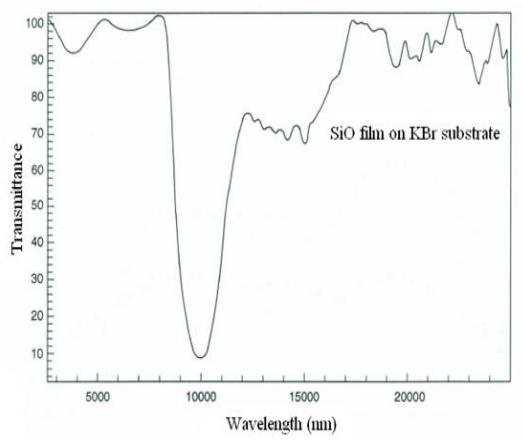

Figure 2: Infrared spectral transmittance of $\mathrm{SiO}$ film of $\mathrm{KBr}$ substrate

The absorption band that appears around a wavelength of about $10 \mu \mathrm{m}$ lies in the region of the atmospheric window $(8-13 \mu \mathrm{m})$. According to Kirchhoff's law a good absorber is a good emitter. Therefore, large emission is expected around $10 \mu \mathrm{m}$ region. Indeed, it is the infrared selective property of $\mathrm{SiO}$ film that is required for the passive cooling of a surface. Therefore, for optimum performance the absorption at $10 \mu \mathrm{m}$ must be as high as possible. The absorption increases as the thickness of the $\mathrm{SiO}$ layer increases. Initially, the transmittance of the $\mathrm{SiO}$ film decrease very rapidly with the film thickness. However, once the thickness of about $1 \mu \mathrm{m}$ is reached then the decrease of the transmittance with thickness declines and becomes very low. It may be noted that with thickness between $1 \mu \mathrm{m}$ to $1.5 \mu \mathrm{m}$, the transmittance decreases only by less than $1 \%$. Therefore, the thickness of $\mathrm{SiO}$ layer of $1 \mu \mathrm{m}$ was used in the present work. The reflectivity of the system on either side of the atmospheric window should be close to $100 \%$ due to Al layer used in the system. The radiative cooling measurements of $\mathrm{SiO} / \mathrm{Al} / \mathrm{glass}$ system were carried out from mid November to mid January. Normally, the weather is a bit rainy during this part of the year and the climatic conditions were therefore far from ideal. The experimental setup was placed on a flat roof in such a way that the plate was freely radiating. Temperature measurements of the surface of the panel and of ambient were continuously monitored with an accuracy of $0.1{ }^{\circ} \mathrm{C}$ on a time base recorder. The results obtained on different dates are reproduced in figures 3-9.

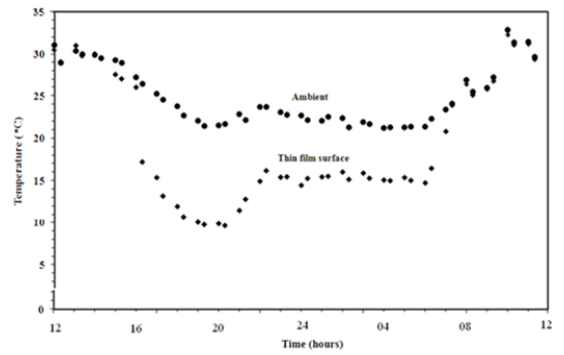

Figure 3: On-line measured temperature of film surface and ambient: November-I 

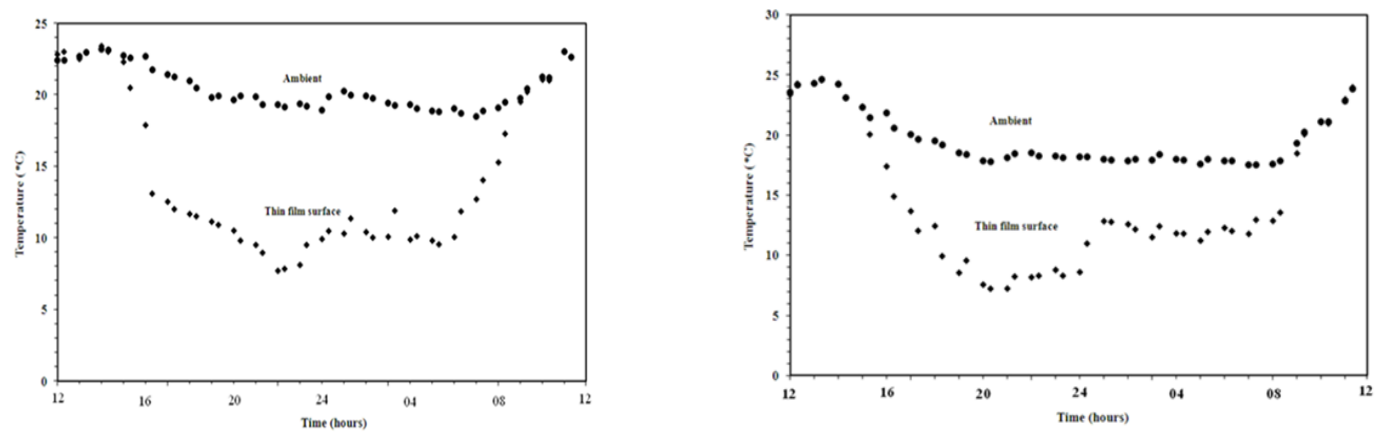

Figure 4, 5: On-line measured temperature of film surface and ambient:

November-II, December-I

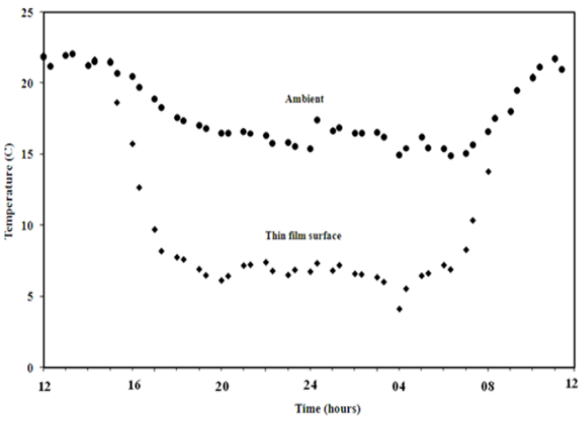

Figure 6: On-line measured temperature of film surface and ambient: December-II

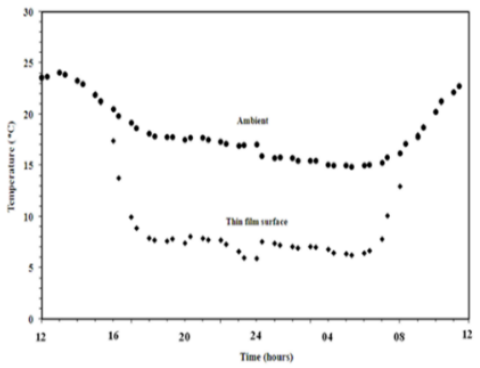


Ahmad, I. and Khawaja, E. E.

Figure 7: On-line measured temperature of film surface and ambient: DecemberIII

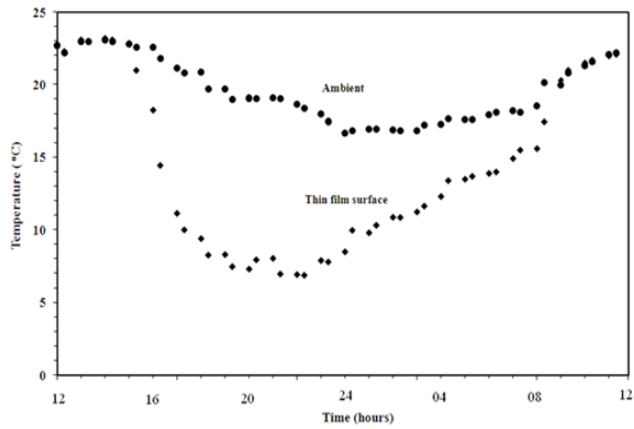

Figure 8: On-line measured temperature of film surface and ambient: January-I

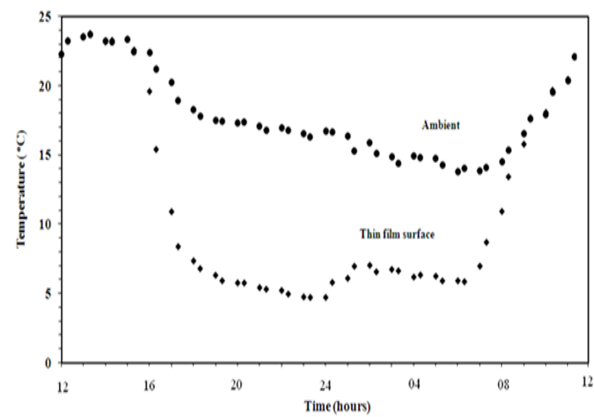

Figure 9: On-line measured temperature of film surface and ambient: January-II

Each of the figures shows two plots corresponding to (a) thin film surface temperature versus time and (b) ambient temperature versus time. It may be noted that starting time measurement was taken at 12 o'clock in the afternoon. This particular time scale was chosen so that the continuity of the cooling cycle (from sunset to sunrise) could be easily visualized from the plots. It can been seen from these figures (3-9) that due to the presence of film surfaces the radiative cooling effects takes place at night. Moreover, the film surfaces are cooler by about $12{ }^{\circ} \mathrm{C}$ as compared with the ambient.

\section{Conclusion}

The spectral transmission in infrared region for $\mathrm{SiO}$ film on $\mathrm{KBr}$ substrate was studied. By testing the spectral selective radiative materials ( $\mathrm{SiO} / \mathrm{Al} / \mathrm{glass})$, it was found that the temperature of $\mathrm{SiO}$ surface $12{ }^{\circ} \mathrm{C}$ below the ambient could be achieved. However, it was observed that for better efficiency of coatings (a) skies should be clear (b) humidity in the air should be as low as possible and (c) the background glow from the streetlights must be at the lowest level possible. Therefore, it is expected that cooling of the surface will be far more efficient in a desert. 


\section{References}

[1] Raman A. P., Anoma M. A., Zhu L., Rephaeli E. and Fan S., Passive radiative cooling below ambient air temperature under direct sunlight. Nature. 2014;515:540-544.

[2] Liu W., Zhang J., Lei B., Ma H., Xie W., Hu H., Ultra-directional forward scattering by individual core-shell nanoparticles. Opt. Express. 2014;22, 16178-16187.

[3] Segev M., Silberberg Y., Christodoulides D.N., Anderson localization of light. Nat. Photonics. 2013;7,197-204.

[4] Xu X., Vignarooban K., Xu B., Hsu K., Kannan A. M., Prospects and problems of concentrating solar power technologies for power generation in the desert regions, Renew. Sustain. Energy Rev. 2016;53, 1106-1131.

[5] Bathgate S.N. and Bosi S.G., A robust convection cover material for selective radiative cooling applications. Sol Energy Mate Sol Cells. 2011;95(10):2778-2785.

[6] Hossain, M. M., Jia B. and Gu M, A metamaterial emitter for highly efficient radiative cooling. Adv. Opt. Mater. 2015;3:1047-1051.

[7] Tso C.Y., Chan K.C. and Chao C.Y.H., A field investigation of passive radiative cooling under Hong Kong's climate. Renewable Energy, 106: 5261(2017).

[8] Atwater H.A., Polman A., Plasmonics for improved photovoltaic devices Nat. Mater. 2010;9,205-213.

[9] Mingke H., Gang P., Lei L., Renchun Z., Junfei L. and Jei J., Theoretical and experimental study of spectral selectivity surface for both solar heating and radiative cooling, Int J Photoenergy. 2015,1-9. Available from: http://dx.doi.org/10.1155/2015/807875.

[10] Granqvist G.C. and Hjortsberg A., Radiative cooling to low temperature: general considerations and application to selectively emitting $\mathrm{SiO}$ films. $J$ Appl Phys. 1981;52:4205.

[11] Tazawa M., P. Jin and Tanemura S., Thin film used to obtain a constant temperature lower than the ambient. Thin Solid Films. 1996; 232:281-282.

[12] Mouhib T, Mouhsen A, Qualim EM, Harmouchi M, Vigneron JP, Defrance P. Stainless Steel/Tin/Glass Coating as Spectrally Selective Material for Passive Radiative Cooling Applications, Opt Mater. 2009;31, 673-677.

[13] Wheeler M.S., Aitchison J. S., Chen J. I., Ozin G. A., Mojahedi M., Infrared magnetic response in a random silicon carbide micropowder. Phys. Rev. 2009;B79:073103.

[14] Muntasir Md. H. and Gu M., Radiative Cooling: Principles, Progress, and Potentials, Adv. Sci. 2016;3,1500360. DOI: 10.1002/advs.201500360

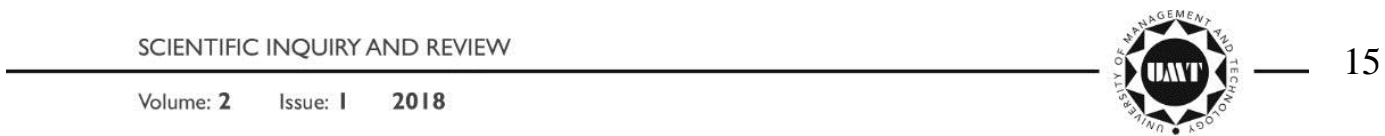

